Sabine Verhulst

\title{
L'itinéraire sensible d'un Brugeois éclairé: le Grand Tour d'André Jacopssen
}

\section{Un BRUGEOIS AUTODICACTE QUI REVE DU GRAND TOUR...}

Lorsqu'il quitte sa Bruges natale au mois d’avril 1821, André Jacopssen, alors âgé de 28 ans, s'apprête à entamer un «Grand Tour » qui le conduira en France, en Suisse, dans l'Italie péninsulaire et en Sicile. Ce voyage qu'il accomplira en compagnie d'un ami brugeois, le baron Brunon-Désiré de Heere avec lequel il avait fixé rendez-vous à Florence au mois de novembre de la même année, durera exactement deux ans, jusqu'au printemps de l'année 1823.

André Jacopssen a consigné souvenirs et impressions de son «Grand Tour » dans sept cahiers autographes, conservés aux Archives de l’État à Gand en Belgique. Les cinq cahiers qui relatent le voyage en Italie ainsi que le cahier sicilien ont été publiés pour la première fois sous le titre d'Itinéraires d'un Brugeois en Italie et en Sicile (1821-1823). ${ }^{1}$ Le journal est écrit à la première personne et suit dans l'ensemble l'ordre chronologique du voyage. D’ordinaire, Jacopssen indique la date de ses étapes et de ses petites excursions, mais dès qu'il s'établit pour un séjour plus ou moins prolongé dans des villes comme Florence, Rome, Naples, Catane ou Palerme, il oublie le calendrier et ne fournit plus aucun renseignement d'ordre chronologique. Les notes recueillies dans ses carnets ne fournissent aucun renseignement sur le voyage du retour, après son deuxième séjour romain. Il est probable qu'après l'hiver romain, au printemps 1823, il soit rentré directement dans son pays, à l'époque le Royaume des Pays-Bas, création du Congrès de Vienne qui devait servir d'Etat-tampon et dissuader les exaltations françaises.

Aîné d'une fratrie de quatre enfants, André Jacopssen naquit en 1793 dans une vieille famille de rentiers originaire de Zélande qui s’était enrichie au commerce du drap et dont les terres s'étendaient entre Bruges et Gand. ${ }^{2}$ Comme d'autres propriétaires terriens, les Jacopssen furent victimes de spoliations suite à l'occupation française et la famille fut astreinte à de sévères restrictions, à telle enseigne qu'il fallut l'héritage d'une tante pour

\footnotetext{
${ }^{1}$ André Jacopssen, Itinéraires d'un Brugeois en Italie et en Sicile (1821-1823), S. Verhulst éd., Genève, Droz, 2008.

${ }^{2}$ Pour la biographie de l'auteur voir l'«Abrégé chronologique de la vie d'André Jacopssen» dans A. Jacopssen, éd. cit., p. XXXII-XL.
} 
qu'André, orphelin de père et responsable de la gestion des affaires de famille depuis 1809, pût réaliser un rêve qui lui chevillait au corps : entreprendre un Grand Tour. ${ }^{3}$

André Jacopssen eut une première instruction plutôt superficielle et aléatoire, et même si ses parents se chargèrent en partie de son éducation, il fut essentiellement autodidacte. Né en Flandre dans une famille bourgeoise durant la période de l'occupation française, il avait, comme il seyait aux élites de l'époque, une bonne maîtrise du français. Il s'essaya à l'écriture autobiographique tant en français qu'en flamand et rédigea ses notes de voyage en français. Touche-à-tout et lecteur omnivore, André Jacopssen était un homme érudit animé d'une grande curiosité, comme en témoignent ses notes de voyage.

Cet article s’interroge sur la nature de la « sensibilité » de Jacopssen telle qu’on peut la reconstruire à partir de son journal de voyage et se propose d'examiner les différents domaines auxquels sont liées les sensations plus ou moins intenses dont fait état notre « touriste » brugeois et quelles sont les circonstances dans lesquelles elles surgissent.

Esquissons d'abord brièvement les grandes étapes de l'itinéraire italien de Jacopssen. Après avoir passé les mois d'été et une partie de l'automne 1821 en Suisse où il est subjugué par la beauté des montagnes et des lacs qu’il parcourt de préférence à pied, il entre en Italie par le mont Cenis et le pas de Suse, pour se retrouver à Turin le 29 octobre 1821. Jacopssen explore tout le versant tyrrhénien de la péninsule jusqu'à Amantea en Calabre. Il passe de Turin à Plaisance, Parme, Bologne, Modène et Florence d'où il fait un saut à Gênes, faisant étape à Lucques, Carrare et, sur le chemin du retour pour Florence, à Pise, Livourne et Sienne. Il quitte Florence le 5 février 1822 pour Rome où il effectue un premier long séjour du 11 février au début du mois de juillet. Du 3 juillet au 11 septembre il s'établit à Naples d’où il explorera Pompéi, Herculanum et le Vésuve. Le voyage se poursuit en bateau : le 12 septembre 1822 il embarque pour la Sicile en faisant escale entre autres à Amalfi, Paestum et à Stromboli. Le 18 septembre, il débarque à Messine d'où il entame un circuit qui lui fera découvrir quasi tout le pourtour de l'île, de Messine à Syracuse, et de Licata à Palerme. Il visitera aussi quelques sites plus à l'intérieur du pays, comme la zone volcanique de l'Etna et

\footnotetext{
${ }^{3}$ Pour la définition et l'évolution du Grand Tour comme institution européenne voir Jean Boutier, Le Grand Tour: une pratique d'éducation des noblesses européennes (XVIe-XVIIIe siècles), 2004, dans http://halshs.archives-ouvertes.fr/halshs-00006836, dernière consultation le 4 août 2011. Pour la tradition du voyage en Italie voir Attilio Brilli, Le voyage d'Italie, Paris, Flammarion, 1989 ; Idem, Il viaggio in Italia. Storia di una grande tradizione culturale, Bologna, Il Mulino, 2006.
} 
les vestiges archéologiques de Palazzolo (Acreide). Le 22 octobre il quitte Palerme pour Naples. A l'arrivée, le 25 octobre, il assiste de la mer à l'une des plus spectaculaires éruptions que le Vésuve a connues durant le XIXe siècle. Jacopssen passera l'hiver de 1822-1823 à Rome. C’est au cours de ce deuxième séjour romain qu'il fera la connaissance de Giacomo Leopardi, avec lequel il se liera d'amitié. ${ }^{4}$ Des lettres que s’échangeront les deux hommes, demeurent des traces qui relèvent de la poétique du grand poète et qui mettent en exergue la thématique de la sensibilité. Au mois de mars il reprend la route du Nord et regagne Bruges en avril 1823.

\section{LE JOURNAL : CHOSES VUES ET SENSATIONS PASSEES}

Le voyage de Jacopssen est un document «brut » qui présente les imperfections inhérentes à ce genre de témoignages non destinés à la publication, surtout du point de vue de la rédaction et de la correction de la langue. Jacopssen était un homme qui avait des lettres, mais il n’était pas écrivain. Son écriture est celle d'un dilettante éclairé; sa syntaxe est parfois bancale, son lexique imprégné de régionalismes et ses notes portent les traces de tournures flamandes et italiennes.

En écrivant un journal de voyage, André Jacopssen ne faisait que respecter ce qui était en réalité une obligation, voire une contrainte, codifiée par la tradition séculaire des «arts de voyager » ou « artes apodemicae » ${ }^{5}$. Les quelques remarques autoréférentielles disséminées dans le journal de Jacopssen sont révélatrices du caractère fondamentalement ambigu de cet exercice : le fait de tenir un journal de voyage est présenté d’une part comme une évidence, mais le journal est ressenti en même temps comme une entrave à la découverte et à la libre gestion de l'emploi du temps. Ce tiraillement même est un topos de la littérature de voyage. Un premier passage métapoétique clôt la description de la ville de Parme. Après avoir évoqué le baptistère, le théâtre et la Bibliothèque Palatine, dont il a pu admirer les volumes précieux qu’il avait consultés, Jacopssen s’attarde sur «le plaisir inimaginable» que lui causèrent les délices vespérales des jardins du Palais Ducal. Les jardins représentent la quintessence du

\footnotetext{
${ }^{4}$ Sur cette rencontre voir Sabine Verhulst, «André Jacopssen e Giacomo Leopardi fra testi e contesti del Grand Tour», in Studi e problemi di critica testuale, 76 (2008), p. 187-219.

${ }^{5}$ Voir, entre autres, Friedrich Wolfzettel, Le discours du voyageur. Pour une histoire littéraire du récit de voyage en France, du Moyen Age au XVIIIe siècle, Paris, PUF, 1996 ; Justin Stagl, Un système de littérature normatrice des voyages au XVIe siècle, in Les guides imprimés du XVIe au XXe siècle. Villes, paysages, voyages, G. Chabaud, É. Cohen, N. Coquery, J. Penez éds., Paris, Belin, 2000, pp. 37-44 ; Idem, A History of Curiosity. The Theory of Travel 1550-1800, Oxon, Routledge, 1995-2006 London \& New York digital Printing, 2004.
} 
bonheur que la découverte de Parme lui inspire. Ne désirant pas s’épuiser à décrire par le menu les charmes des jardins et de la ville, il renvoie aux guides existants (« pour le reste voir l’itinéraire ») en précisant aussitôt:

La ville de Parme à elle seule demanderait plus de papiers que ce volume, il faut y renoncer car le temps pour décrire tout ce qu'il y a de remarquable serait immense. [...] Le temps que l'on met à décrire tout ce que l'on voit est considérable, c'est autant de perdu pour la jouissance. Jouir et profiter de ce que l'on a un moment à voir, doit être la maxime d'un voyageur qui n'a pas de temps à perdre et qui se trouve [dans le sein] au sein d'un pays mille fois décrit. (p. 20)

On trouve le même dilemme dans les annotations rédigées au début du séjour romain. Jacopssen était arrivé dans la ville Éternelle le 11 février 1822. Si d’après lui la toute première impression de Rome est positive, dès que l’on a passé Place du Peuple et qu'on entre dans le cœur de la ville, on est déçu par l'étroitesse des rues, le manque d’homogénéité architecturale, la disgrâce du style baroque (« architecture fatiguée, laborieuse et de goût détestable du siècle des Borromini, Valvassori ») et les stucs décolorés. Jacopssen impute la cause de cette déception à nul autre qu’à Dupaty:

Je fus d'autant plus désappointé que d'après la description démesurément brillante de Dupaty sur Rome, je m'attendis aux magiques sensations que cette ville allait faire naître dans mon esprit $[\ldots]($ p.91)

Notre Brugeois prend toutefois la peine de nuancer son propos et précise aussitôt après avoir visité plus attentivement la ville, il se laissa moins emporter par l' imagination. Il écrit:

Je la considérai sous un point de vue plus sain, moins prévenu et avec l'imagination moins exigeante que j'y portai d'abord. C'est un fait reconnu par bien des voyageurs que Rome attache progressivement par le séjour que l’on y fait. (p. 91)

Jacopssen dialogue indirectement avec toute la tradition du voyage en Italie telle qu'elle apparaît dans le témoignage écrit, des instructions " apodémiques » aux formes diverses de récits de voyage littéraires ${ }^{6}$. Devant l’ «immensité des objets dont Rome fourmille » il prend le parti suivant :

Je comptais abandonner ici ces faibles et incorrectes notes, mais pour me conformer à mon premier plan, à l'usage et à des considérations majeures en quelque sorte, dont une des principales serait le reproche de négligence, je vais donc jeter ici sur le papier le petit nombre de remarques ou d'annotations que le peu de loisirs me permettront de faire. Elles

\footnotetext{
${ }^{6}$ Les représentations de l'Italie et de la Sicile telles qu'elles prennent forme dans les Itinéraires de Jacopssen sont bien évidemment conditionnées par ses lectures, qui comprenaient aussi bien des guides et des récits de voyage que des écrits philosophiques et des romans, et encore par l' iconographie du " Grand Tour » qui avait contribué à façonner le mythe visuel de l'Italie. Voir à ce propos A. Jacopssen, éd. cit, pp.XXVIII-XXX et S. Verhulst, art.cit., p. 201-218.
} 
me rappelleront, outre mes sensations passées et des petites particularités dont je veux me ressouvenir. Je fais ici ces remarques afin que, si ces papiers tombaient par malheur en mains étrangères, l'on soit assez généreux de croire que j’ai tiré un tant soit peu plus de fruit de mes voyages que ces écrits pourraient le faire supposer » (p.91-92)

Jacopssen se trouve confronté à ce que Emmanuelle Hénin appelle «le vertige de l'énumération» qui ne semble laisser au voyageur que le choix rhétorique entre «le refuge» de la prétérition» et «la description plus détaillée d’un objet, arraché à la liste par l'attention particulière du locuteur» ${ }^{7}$.

Deux pages plus loin, il revient sur la question :

Nous allons sans suivre aucun ordre, aucune suite, parler de cette ville et de ses monuments, ainsi que quelques idées ou souvenirs ou plaisirs ou peines qu'ils m'ont suggérés. Dieu me garde de tous les objets que j'aurais vus ou pas vus, toutes les galeries, toutes le statues, les 346 églises, les 180 à 200 palais, etc. (p. 94)

Dans un premier temps il semble opter pour une organisation thématique du journal de bord romain et commence par un chapitre consacré aux obélisques. Suit la description systématique d’une dizaine d'églises précédée d’une réflexion critique sur l'évolution des mœurs en matière de religion. Mais après la description des Carceri Nuove, il abandonne définitivement son plan en traitant pêle-mêle différents sujets. Quelques pages plus loin il fait une nouvelle mise-au-point:

En relisant les précédentes notes avec leur pitoyable style et [le] peu d'idées neuves avec lesquelles elles sont rédigées, je m’aperçois que ce serait le comble de la vanité et de l'aveuglement que de les continuer. Changeons encore ici de plan, tâchons d'employer moins mal nos heures. (p. 116)

Une des raisons pour lesquelles il décide de ne pas abandonner son journal est bel et bien le désir de conserver une trace de ses « sensations passées ». Si l’idée des sensations reprend la thématique chère à Dupaty ${ }^{9}$, il faut préciser que le fait de privilégier l’information fournie par les sens était une exigence déjà codifiée dans certains manuels de voyage antérieurs aux Lettres sur l'Italie de Dupaty. Jean-Cyrille Sow renvoie par exemple au Manuel de l'étranger

\footnotetext{
${ }^{7}$ Emmanuelle Hénin, «Rome, un lieu commun ? Usage et usure du topos dans les récits des voyageurs français à Rome au XVIIe siècle», in Revue d'Histoire Littéraire de la France, 104 (2004)3, p. 597-619 ; p. 607.

${ }^{8}$ Il n’est évidemment pas exclu que Jacopssen ait pu caresser l'idée d'écrire un voyage d'Italie destiné à la publication.

9 Charles Mercier-Dupaty, Lettres sur l'Italie en 1785, Paris, De Senne, 1788; I, p. 303: «D'autres rapporteront de Rome des tableaux, des marbres, des médailles, des productions d'histoire naturelle ; moi, j'en rapporterai des sensations, des sentimens et des idées ; et sur-tout les idées, les sentimens et les sensations qui naissent au pied des colonnes antiques, sur le haut des arcs de triomphe, dans le fond des tombeaux en ruine, sur les bords marécageux des fontaines » (Lettre LXIV).
} 
qui voyage en Italie de Jacques-Dominique Cassini paru à Paris en 1778 et qui comporte les conseils suivants:

N'allez point, observateur aveugle et timide, consultant sans cesse votre livre, demander à chaque pas à votre auteur : cela est-il beau ? C'est vous, au contraire, qui devez vous prononcer le premier. [...] C'est un principe général et incontestable qu'auparavant de juger d'après les règles, il faut toujours juger par le sentiment, c'est-à-dire d'après le premier effet et l'impression plutôt naturelle que raisonnée que fait sur vous l'objet de votre examen ${ }^{10}$.

Avec Stendhal d'ailleurs, on reste dans le même esprit, lorsque le $1^{\mathrm{er}}$ juillet 1813 celui-ci déclare dans son journal, après avoir chanté les louanges du Voyage de Misson:

Un journal de voyage doit être plein de sensations, un itinéraire en être vide. [...] Le mélange de la sensation avec l'indication est détestable et diminue infiniment le plaisir du voyageur qui se trouve en présence de ce qu'un autre homme a senti, au lieu d'être livré à son propre sentiment ${ }^{11}$.

Le document «privé » que nous a laissé André Jacopssen oscille en permanence entre deux exigences, celle de dresser le catalogue des choses vues d'une part et de conserver une trace des sensations personnelles que la vue de ces choses lui a inspirées de l'autre. Le résultat de ce choix se traduit inévitablement par un texte hybride.

\section{SENSIBILITE ET DISCOURS ENCOMIASTIQUE}

D’après les notes de Jacopssen, plusieurs domaines furent générateurs d'élans d'enthousiasme et de projections «sensibles» personnelles, parmi lesquels les Beaux-Arts, les ruines antiques, les Sciences de la Nature, les Musées d’Histoire Naturelle et la philanthropie occupèrent une place de choix.

L'on retrouve toutefois dans le journal de Jacopssen des traces de cette «encomiastique aux motifs fixés d'avance» qui, comme l'a bien démontré Emmanuelle Hénin ${ }^{12}$, caractérisait le récit du voyageur depuis le XVIIe siècle et qui aimait à «mettre en scène perpétuellement l’étonnement du locuteur».

\footnotetext{
10 Jean-Cyrille Sow, Les guides d'Italie du XVIIIe siècle et la formation d'une critique française de l'art italien moderne, in Les guides imprimés du XVIe au XXe siècle. Villes, paysages, voyages, textes réunis et publiés par G. Chabaud, É. Cohen, N. Coquery, J. Penez éd., Paris, Belin, 2000, p. 155-166 ; p. 165.

${ }^{11}$ Stendhal, Journal (1801-1817), dans Stendhal, Oeuvres intimes, I, V. Del Litto éd., Paris, Gallimard, 1981, p. 878.

${ }^{12}$ E. Hénin, «Rome, un lieu commun ? ...», p. 605. E. Hénin appréhende Rome comme topos du récit de voyage au XVIIe siècle, mais ses observations sur le discours encomiastique me semblent valables pour le récit de voyage en Italie en général.
} 


\section{LES BEAUX-ARTS}

Les Beaux-Arts dans leur ensemble sont une source continuelle de ravissement pour notre voyageur brugeois. Il visite les musées, les galeries privées, comme la galerie de tableaux du Prince Poniatowski à Rome, mais aussi de nombreux ateliers d'artistes. La visite à l'atelier du sculpteur Cincinnato Baruzzi (1796-1871), élève préféré de Canova, suscite en lui émerveillement et tendresse à la vue de trois sculptures exposées. Jacopssen ne tarit pas d'éloges sur la façon dont Baruzzi manie le ciseau et sur la grâce de l'exécution. Il décrit les trois statues par le menu : une nymphe, une autre représentant Amour et enfin une sculpture de Psyché. Jacopssen assistera également à une séance commémorative d’Antonio Canova, mort le 13 octobre 1822, à l’Accademia delle Belle Arti (ou di San Luca) le 31 janvier 1823. Notre Brugeois était décidément un enfant de son temps et son engouement pour l'art néoclassique est tangible dans l'évocation circonstanciée de sa visite à l'atelier du peintre Vincenzo Camuccini (1771-1844), qui était alors au faîte de la gloire. Camuccini était célèbre pour le dessin, en particulier pour ses copies de Raphaël, le peintre préféré de Jacopssen. Jacopssen a un faible pour les dessins à sujet mythologique de Camuccini; l'admiration pour le remarquable coup de crayon du peintre n'empêche toutefois pas quelque réserve sur sa façon de manier la couleur.

Mais tous les styles n'ont pas l'heur de plaire à notre voyageur, qui n'arrête pas de distribuer bons et mauvais points tout au long de son journal. L'art baroque, et en particulier l'architecture et la sculpture, ne trouvent aucune grâce à ses yeux. Les œuvres de Borromini sont dénigrées, comme par exemple la Cappella dei Re Magi, incorporée dans la façade du Palais de Propaganda Fide, qu'il décrit ainsi :

Le grand bâtiment de la Propaganda Fide est le célèbre collège institué pour la propagation de la foi en 1622. La façade est du Bernin, simple et sage. L'église faisant face à la rue latérale est une des plus extravagantes compositions sorties de la tête du Borromini. Sous le rapport de l'élévation extérieure, on y voit une suite de niches et lignes courbes sans aucune règle, de manière que cette façade n'offre quasiment ni commencement, ni milieu, ni repos, ni fin. (p. 242-243)

Jacopssen est en général plus indulgent pour le Bernin, mais lorsqu’il évoque la statue de Louise Albertoni dans San Francesco a Ripa il écrit :

Cette sainte est couchée de son long et mourante, la tête et les mains sont très belles, mais les draperies sont lourdes et empesées comme beaucoup d'autres de ce grand artiste. (p. 232) 
Toutefois c’est l'art médiéval qui lui inspire les jugements les plus dépréciatifs. Deux exemples. Le dôme de Modène, pur chef-d'œuvre de l'architecture romane est disqualifié d'une formule lapidaire : « la cathédrale est un gothique de mauvais genre » (p. 21). Comme le terme «art roman » n'était pas encore passé dans l'usage ${ }^{13}$, l'on ne s'étonnera guère que Jacopssen confonde les deux styles. Jacopssen éreinte en outre les bas-reliefs représentant les Scènes de la Passion qui ornent le jubé, il n’y voit que maladresse et trouve l'ensemble des sculptures plutôt risible ${ }^{14}$.

Même jugement intransigeant pour la cathédrale de Lucques. Le Duomo, dédié à saint Martin, est aujourd'hui célèbre pour sa magnifique façade ornée de trois galeries à colonnades. Le vestibule est orné de reliefs romans. Pour Jacopssen, « ces colonnes [...]toutes diversement contournées, incrustées, cannelées, sculptées et taillées en vis, etc., avec basreliefs, fleurs, dessins, en marbres de couleur [...]» forment « un travail plus curieux, plus étonnant que choisi et bon. » (p.32-33).

\section{LES RUINES ANTIQUES}

Pour ce qui est des monuments et des ruines antiques, les vestiges plaisent surtout à Jacopssen dans la mesure où ils sont recouverts de végétation sauvage et qu'ils se métamorphosent ainsi en éléments du paysage. Le Panthéon par exemple pâtit de l’environnement urbain délabré dans lequel il est situé (« ses vilaines grilles modernes et les habitations malpropres dont il est entouré » p. 117) ; le Colisée en revanche «offre un coup d'œil ravissant du côté où il est couvert d'arbres, d'arbustes et de plantes sauvages» (p. 117). La contemplation des grands ouvrages comme les aqueducs est une source inépuisable de joie empreinte de mélancolie :

Aujourd'hui c'est un coup d'œil à la fois superbe et mélancolique que de voir la campagne jonchée de grandes lignes d'arcades qui sillonnent encore çà et là des champs cultivés ou sauvages. Tantôt une dizaine, vingtaine ou centaine de ces portiques qui se détruisent

\footnotetext{
${ }^{13}$ Voir l'entrée «roman» (adjectif) dans le Dictionnaire historique de la langue française, Paris, Dictionnaires Le Robert, 1992.

14 «Ce sont quelques traits de la passion de J.-C. On y voit d'abord la Cène, les douze apôtres debout devant une table dans des attitudes les plus forcées. [...] Plus loin, c'est la trahison de Judas qui vient embrasser son maître, encore dans le Jardin des Olives tenant son livre de prières à la main. Dans la position la plus grotesque, élevé sur la pointe des pieds, il fait tous ses efforts pour atteindre la joue de J.-C. Ces deux figures font des grimaces drôles. [...] Ailleurs c'est la flagellation; plus loin encore c'est Jésus qui porte la croix. Toutes ces figures sont de la plus risible expression. Là J.-C. est un géant, quelques juifs, des pygmées et quelques apôtres, des hommes. La croix que Jésus porte a la longueur de son bras, etc. » (p. 21-22).
} 
miette à miette pour ainsi dire, car l'homme étant las de détruire ces solides masses, le temps seul doit effacer le reste. (p. 131)

Comment ne pas reconnaître ici l'écho du discours sur les ruines dans les écrits de Bernardin de Saint-Pierre et de Chateaubriand qui tous deux distinguent les ruines « ouvrage du temps » de celles qui sont « l'ouvrages des hommes » ${ }^{15}$.

C’est donc dans leur sillage et dans celui de Corinne Madame de Staël que Jacopssen revisite le topos du spectacle dynamique des ruines ${ }^{16}$ et adhère à la stéréotypie néoclassique qui s’était développée autour de l’idée de mélancolie. La découverte du site abandonné de Paestum entraîne une description jubilatoire de ce qu'il appelle « un éloquent tableau de la nature ». Jacopssen se complaît à décrire par le menu le foisonnement des plantes sauvages qui envahissent les fragments de marbre et les colonnes qui jonchent le sol. Les modèles «botaniques» des Études de la nature de Bernardin de Saint-Pierre et des Rêveries du promeneur solitaire de Rousseau inspirent ainsi à Jacopssen des fragments plutôt réussis autour de la terminologie des végétaux, par exemple à l'occasion d'une visite du Palatin.

\section{LES SCIENCES DE LA NATURE ET LES MUSEES D’HistOIRE NATURELLE}

Un autre domaine qui passionne André Jacopssen est celui des Sciences Naturelles. Les visites aux Musées d'Histoire Naturelle donnent invariablement lieu à des descriptions circonstanciées des collections. De même, l’observation sur le terrain de phénomènes géologiques et minéralogiques est-elle toujours à l'origine d'évocations enthousiastes, par exemple lorsqu'il explore les sites volcaniques du Vésuve, du Stromboli et de l’Etna. L'autodidacte André Jacopssen était animé d'une authentique soif de connaissances encyclopédiques et les cabinets de curiosités scientifiques et autres «Wunderkammer » donnent lieu dans son journal à des énumérations fébriles comme s’il voulait reproduire par l'écrit l'accumulation des objets exposés.

La description de l'étrange capharnaüm qu'était le cabinet de curiosités d'Athanase Kircher installé au Collegio Romano à Rome est d'autant plus précieuse que notre Brugeois fait état de la collection telle qu'elle était en 1822, c’est-à-dire quelques années avant sa dispersion définitive ${ }^{17}$. Jacopssen précise que l’on voit au Collegio Romano peu de choses

\footnotetext{
${ }^{15}$ Voir à ce propos S. Verhulst, art. cit., p. 215-218.

${ }^{16}$ Voir Renzo Negri, Gusto e poesia delle rovine in Italia fra il Sette e l'Ottocento, Milano, Meschina 1965 et S. Verhulst, art. cit., p. 210-218.

${ }^{17}$ Une partie des objets est à la base de la collection de l'actuel Museo Nazionale Preistorico Etnografico Luigi Pigorini à Rome.
} 
de Kircher, mais il fait malgré tout état d’un nombre encore assez impressionnant de petits objets. Notre voyageur est ainsi intrigué par la collection d’objets érotiques anciens réunie par le père Jésuite, à telle enseigne que la visite lui inspire une brève digression sur ce qu'il appelle la «sensibilité » ou « sixième sens » tel qu'il est défini dans l'Histoire naturelle de Buffon, et qui n’est autre que la voluptée ${ }^{18}$. À Catane, il exulte en visitant le Musée fondé au XVIIIe siècle par l'archéologue et mécène Ignazio Paternò, prince de Biscari : il s’agit d'un témoignage d’autant plus intéressant sur l'état du Musée et de la Galerie d’Histoire Naturelle qu'abritait le Palazzo Biscari au début du XIXe siècle, qu'en 1930 les collections seront définitivement transférées au Castello Ursino ${ }^{19}$.

Nombreux sont les passages où Jacopssen s'émerveille devant l'immense musée scientifique à ciel ouvert qu'est la nature. Il aime à décrire les strates géologiques, les divers phénomènes minéralogiques, les coulées de lave solidifiées, les falaises crétacées et la végétation toujours changeante. Jacopssen avait parcouru le Jura suisse d'un regard façonné par la leçon «sublime » de Buffon. Il voyait s’ouvrir devant lui les archives tenues par une nature s'exprimant dans des montagnes-océans, faites d' « énormes bancs de coquillages». Chaque montagne d'Italie rappelle ainsi à Jacopssen que la croûte terrestre et les montagnes sont modelées par le travail millénaire des eaux. Notre voyageur se sentait comme projeté dans la perspective immémoriale et fascinante de l'histoire du globe terrestre ${ }^{20}$.

\section{UNE SENSIBILITE DE PHILANTHROPE}

La sensibilité philanthropique de notre voyageur brugeois se manifeste dans toute son intensité lors de visites aux hôpitaux, aux prisons et aux instituts de bienfaisance. Je me contenterai de signaler deux exemples, tous deux empruntés au cahier napolitain. À Naples Jacopssen visite l'Hôpital des Incurables. Comme toujours lorsqu'il remémore ce genre de visites, il ne se contente pas de dépeindre ce qu'il a vu, comme le nombre et le sexe des patients, les pathologies dont ils souffrent, le type de soins qui leur sont administrés, les

\footnotetext{
${ }^{18}$ Dans le Discours sur la nature des oiseaux Buffon explique l'ordre des sens, c'est-à-dire des «sensations dominantes » chez les différentes espèces animales (quadrupèdes, oiseaux) et l'homme : toucher, goût, vue, ouïe, odorat. Il précise qu' " il y a un sixième sens qui, quoique intermittent, semble, lorsqu'il agit, commander à tous les autres, et produire alors les sensations dominantes, les mouvements les plus violents, et les affections les plus intimes ; c'est le sens de l'amour [...].» Voir Buffon, Oeuvres, S. Schmitt éd., Paris, Gallimard, 2007, pp. 1055- 1090; p. 1084.

${ }^{19}$ Pour l'histoire du musée, voir Stefania Pafumi, Museum Biscarianum. Materiali per lo studio delle collezioni di Ignazio Paternò Castello di Biscari (1717-1786), Catania, Almaeditore, 2006.
}

\footnotetext{
${ }^{20}$ Voir S. Verhulst, art. cit., p. 205-206.
} 
équipements, etc., il se concentre aussi sur quelques patients dont il évoque le sort tragique. À l'Hôpital des Incurables - nom qu'il trouve scandaleux puisqu'il ôte tout espoir aux patients qui sont accueillis dans ce lieu - il a vu une jeune fille atteinte d'un cancer foudroyant: l'image de son corps frêle ravagé par la maladie le touche profondément. Jacopssen nous a aussi laissé un témoignage rare sur un autre établissement de charité, l’immense Albergo dei Poveri de Naples, appelé également Reclusorio, destiné à accueillir et à éduquer 8.000 citoyens défavorisés. Le projet architectural démesuré conçu par l'architecte Ferdinando Fuga à la demande de Charles III de Bourbon est resté inachevé ${ }^{21}$. Grâce à Jacopssen nous pouvons nous faire une idée assez précise de la façon dont s’y déroulait la vie quotidienne au début du XIXe siècle. Il n’est autorisé qu'à visiter la section des hommes et est impressionné par l’ordre et la propreté des lieux, en particulier des dortoirs. Il décrit la méthode qui permet aux sourds-muets d'apprendre à articuler.

\section{CONCLUSIONS}

Comme ces quelques exemples tirés de champs différents permettent de le constater, l'âme sensible de Jacopssen aime à résonner au diapason des objets et des phénomènes, parce que ceux-ci invitent l'homme à se projeter dans le Temps et qu'ils lui font prendre conscience du «flux impétueux des générations humaines » se succédant sans fin depuis la nuit des temps. Plus d’une fois il a l'occasion de visiter des sites archéologiques à peine dégagés ou encore partiellement enfouis. Un colombarium fraîchement découvert à Rome en février 1822 met à jour des rangées de niches abritant de nombreux vases funéraires et Jacopssen imagine aussitôt avec tendresse la vie des défunts dont seules les cendres séculaires gardent le souvenir. À Akrai en Sicile, la future Palazzolo Acreide, il assiste aux fouilles menées par le baron Judica : l'ouverture en sa présence d'une tombe qui révèle le squelette d’une femme et un vase étrusque suscite chez lui un sentiment double, où plaisir et tristesse s'entremêlent. Mais sa passion pour l'Histoire peut aussi prendre la forme concrète de l'indignation lorsqu'il constate que les Romains contemporains ne s’intéressent guère à leur passé et qu'il manque des ouvrages récents retraçant l'épopée de la Ville éternelle. Pour rester dans le domaine des livres, Jacopssen est choqué par le sort réservé aux bibliothèques des monastères expropriés par les Français et aux innombrables livres confisqués, puis bradés dans les rues. Il mentionne le célèbre libraire romain De Romanis dont les remises regorgent de livres saisis. Ce qui peine le plus notre voyageur brugeois, c'est lorsqu'il constate le manque total de respect pour ce que

\footnotetext{
${ }^{21}$ Voir Donatella Mazzoleni, Palazzi di Napoli, Venezia Mestre, Arsenale Editrice, 2000, pp. 210-219.
} 
représentent ces ouvrages. Il note avec amertume: «Les éditions antiques, rares, belles, précieuses, toutes passèrent pêle-mêle dans les mêmes balances, on se les jette à la tête par les rues de Rome » (p. 249). Et il se désole en particulier sur les chargements entiers de livres de valeur qui partent pour l’Angleterre.

Le lecteur ne trouvera pas dans le témoignage de Jacopssen les grands tableaux plus ou moins «sensibles» sur l'état ecclésiastique de Rome, sur la vie politique et les mœurs des Italiens, ou sur la population du royaume de Naples, qui font le charme et l'intérêt des Lettres sur l'Italie de Dupaty. Si dans les annotations fébriles et spontanées du Brugeois ces éléments se font jour ici et là par petites touches, les carnets de voyage révèlent avant tout une foi inébranlable dans le progrès des sciences naturelles, conformément à la leçon « encyclopédique » des Lumières, tempérée par une sensibilité oscillant entre une approche subjective de l'expérience esthétique (le goût) et une compassion authentique pour le sort du prochain. La vision de l'Italie qui émane des notes de Jacopssen est le résultat d'une formation peu structurée, mais qui n'en est pas moins solide et nourrie. A travers ses annotations toujours attentives au déroulement matériel du voyage et aux changements de paysage, et en prise directe sur le quotidien, Jacopssen nous a laissé l'image d'un Grand Tour qui reflète la culture d’un lettré flamand du début du XIXe siècle. 